\title{
COMENTARIO AL PROYECTO DE REFORMA CONSTITUCIONAL QUE CREA EL CARGO DE FISCAL ESPECIAL DE ALTA COMPLEJIDAD EN EL MINISTERIO PÚBLICO (BOLETÍN No 9608-07)
}

COMMENTARY TO THE CONSTITUTIONAL REFORM PROJECT THAT CREATES A

SPECIAL HIGH COMPLEX FISCAL POSITION IN THE DISTRICT ATTORNEY OFFICE

(BOLETíN No 9608-07)

COMMENTAIRE AU PROJET DE RÉFORME CONSTITUTIONNELLE

QUI PROPOSE LA CRÉATION DU POSTE DE FISCAL SPÉCIAL DE HAUTE

COMPLEXITÉ DANS LE MINISTÈRE PUBLIC (BOLETÍN No 9608-07)

Leonardo Cofré Pérez*

El presente trabajo analiza el proyecto de reforma constitucional que crea el cargo de Fiscal Especial de Alta Complejidad en el Ministerio Público, presentado al Congreso Nacional mediante mensaje presidencial en el mes de septiembre de 2014. Previamente, se entregan elementos de contexto respecto de la gestión de delitos denominados "complejos" y las iniciativas que se han presentado en nuestro país al respecto. Posteriormente, se presentan algunas opiniones respecto de la importancia de esta reforma, desde el diseño institucional, el derecho constitucional y la política pública de justicia criminal.

\section{INTRODUCCIÓN Y CONTEXTO}

Más allá de los constantes y necesarios debates respecto a los resultados del sistema de justicia criminal chileno, especialmente en relación a los fines perseguidos por el proceso penal, lo cierto es que existe consenso en cuanto a considerar al sistema en un estado de maduración aceptable luego de los primeros años de su instalación ${ }^{1}$. Consecuencia de esto es que para los encargados de la política pública de justicia surge la necesidad de hacerse cargo de nuevos problemas, internos al sistema procesal y externos a él, referidos a la sociedad en su conjunto.

* Abogado. Licenciado en Ciencias Jurídicas y Sociales de la Universidad de Chile. Magíster (C) en Derecho Público por la Universidad de Chile. Profesor asistente Facultad de Derecho de la Universidad de Chile. Correspondencia a: Correo electrónico lcofre@derecho.uchile.cl. Agradezco los aportes de la estudiante de derecho, María Jesús Núñez.

${ }^{1}$ Como evaluaciones de los primeros años del sistema procesal penal, se puede citar por ejemplo DuCE Julıo, Mauricio (2011). "Diez años de la reforma procesal penal en Chile: apuntes sobre su desarrollo, logros y desafíos”. En: Diez años de la Reforma Procesal Penal en Chile, Fuentes, Claudio (Coord.), Santiago: Ediciones Universidad Diego Portales, pp. 23-73. 
Estos nuevos desafíos se manifiestan, por ejemplo, en una revisión y mejora de los mecanismos legales de rendición de cuentas de los organismos encargados de la persecución penal, en la necesidad de perfeccionar los medios de coordinación entre estas instituciones y en definitiva, a través de una modernización del sistema que produciría una persecución más eficaz de los delitos, especialmente de aquellos que representan un esfuerzo más intenso para los órganos que conforman este sistema, que han sido denominados como "delitos complejos" o "de alta complejidad".

Si bien los delitos complejos no representan una gran cantidad de trabajo para el Ministerio Público, son importantes para medir la eficiencia y calidad de la respuesta penal. Sin embargo, se debe reconocer que la categoría es vaga y difusa. ¿Qué debe entenderse entonces por "delitos de alta complejidad"? En términos generales se trata de aquellos casos que poseen una alta connotación social, es decir, se trata de aquellos que causan gran temor, impacto o daño social, y también aquellos que exigen un grado relativamente alto de coordinación, profesionalismo o persistencia en el tiempo, tanto para el Ministerio Público como para los organismos que colaboran con él ${ }^{2}$.

Así, en primer lugar, en cuanto al impacto público del hecho, ese criterio se ha construido simplemente sobre la base del lugar que en la agenda pública tiene o ha tenido un cierto tipo de delito. Se debe reconocer aquí que esa agenda no es rígida, y se determina en gran medida por lo que los medios de comunicación van reconociendo como relevante. En segundo lugar, el delito de alta complejidad se define también porque es capaz de colocar al sistema de justicia frente a desafíos de profesionalización mayores a los que exige la gestión de casos comunes y masivos. Y por último, también se podrían agregar a esta denominación los casos relativos a "enigmas de repercusión colectiva", en los que si bien se podría discutir si existe un contenido jurídico penal, pueden llegar al sistema de justicia en búsqueda de esclarecimiento ${ }^{3}$.

Lo que demuestran estudios comparados en América Latina es que existe una baja capacidad de los sistemas para perseguir eficazmente este tipo de delitos, pues se aprecian pocos resultados exitosos de investigación y condena de estos delitos, especialmente a causa de debilidades de los sistemas judiciales en materia de obtención de prueba. Esto ha llevado al uso masivo de la facultad de archivar este tipo de casos, lo que ha contribuido al problema de una creciente sensación, por parte de la ciudadanía, de impunidad frente a la comisión de estos ilícitos ${ }^{4}$.

${ }^{2}$ Centro de Estudios de Justicia de las Américas (2010). Persecución de delitos complejos. Capacidades de los sistemas penales en América Latina. Santiago: Centro de Estudios de Justicia de las Américas, p. 14.

3 De la BarRa Cousiño, Rodrigo (2010). "Desafíos para la investigación y persecución penal de delitos complejos en países de las Américas. Informe Nacional de Chile”. En Persecución de delitos complejos. Capacidades de los sistemas penales en América Latina, Santiago, Centro de Estudios de Justicia de las Américas, pp. 159-161.

${ }^{4}$ Centro de Estudios de Justicia de las Américas (2010), pp. 43-44. 
Considerando aquello, uno de los principales órganos que se ve tensionado por estas demandas es el Ministerio Público, debido al rol que cumple en el sistema jurídico. En efecto, esta institución fue creada con el objetivo de abolir un sistema penal antiguo, caracterizado por el principio inquisitivo y la falta de imparcialidad del juzgador. Pero también, como explica Roxin, se trata de un organismo que cumple una función de aseguramiento del Estado de derecho, pues al entenderse como un funcionario objetivo de instrucción, se reconoce como un custodio de la ley en que su tarea "consiste en velar, a favor del imputado, porque se obtenga todo material de descargo y porque ninguno de sus derechos procesales sea menoscabado"s.

\section{Propuestas sobre gestión de los delitos de alta complejidad}

De esta forma es que se ha radicado en el Ministerio Público la exigencia de gestionar de forma eficiente los delitos más graves para nuestra sociedad, lo que a su vez genera una serie de problemas de elección y diseño institucional, considerando que los recursos son escasos. Además, el contexto actual en el que se desarrolla esta demanda, en el que han explotado complejas causas que involucran aspectos problemáticos de la relación entre la política y el dinero, hace más radical la discusión respecto a las posibles soluciones ${ }^{6}$. Con todo, en los últimos años en nuestro país se han presentado algunas propuestas, que se mencionarán brevemente a continuación.

Uno de los primeros documentos que menciona la creación de una fiscalía especial para delitos complejos es el "Proyecto de Fortalecimiento del Ministerio Público" para los años 2010-2013. Este texto señala 5 proyectos de perfeccionamiento de la institución, entre los que se encuentra la creación de una fiscalía supraterritorial. Se trata de la instalación de una nueva estructura dentro del Ministerio Público, dedicada a casos de criminalidad compleja. Innova en cuanto a la organización, pues se considera de carácter supraterritorial, a diferencia de las fiscalías regionales, que investigan y persiguen los delitos dentro de un ámbito territorial específico, y en cuanto a gestión y coordinación, pues manifiesta una nueva forma de trabajo interinstitucional permanente y multidisciplinaria ${ }^{7}$.

De acuerdo a la Fiscalía, las características que ese nuevo órgano debería poseer, consisten en: (i) contar con un funcionamiento territorial y autónomo (para garan-

\footnotetext{
${ }^{5}$ Roxin, Claus (1993). "Posición jurídica y tareas del ministerio público". En: El Ministerio Público en el Proceso Penal, AA.VV., Buenos Aires: Ad Hoc, pp. 39-40.

${ }^{6}$ Me refiero a los denominados casos "Penta" y "SQM". Sobre estos casos se puede consultar la página web creada especialmente por la ONG Ciudadano Inteligente para explicarlos, disponible en: $<$ http://casopenta. cl/>. [Consulta: 10 mayo 2015]. Respecto a la presión sobre el Ministerio Público, ver por ejemplo Qué PASA (2015). Acosados. Disponible en: <http://www.quepasa.cl/articulo/politica/2015/04/19-16803-9-acosados. shtml>. [Consulta: 30 abril 2015].
}

${ }^{7}$ Ministerio Público (2009). Proyecto de Fortalecimiento del Ministerio Público 2010-2013, pp. 17-23. 
tizar independencia de los fiscales); (ii) un objetivo de investigación y persecución de delitos de alta complejidad (por ejemplo, casos de corrupción, crimen organizado -especialmente narcotráfico- y lavado de dinero); (iii) un nuevo modelo de trabajo interinstitucional y multidisciplinario: funcionarios de Carabineros y de la Policía de Investigaciones incorporados de manera permanente; y, (iv) que sea diseñado en coherencia con otras buenas iniciativas comparadas y recomendaciones internacionales. Para la fecha de elaboración del Proyecto por parte del Ministerio Público, se había propuesto contar con un personal de 83 nuevos cargos, y se esperaba que esta Fiscalía asumiera aproximadamente el 5\% del total de causas. En cuanto a su estructura, se propuso un organismo de la misma entidad y jerarquía que las fiscalías regionales ${ }^{8}$.

A juicio del Banco Interamericano de Desarrollo ${ }^{9}$, esta propuesta parece adecuada para el problema detectado, sin perjuicio de que sería posible dividir la organización de esta fiscalía supraterritorial con base en el tipo de delitos (por ejemplo, una entidad especializada en narcotráfico), aunque reconoce que ello corresponde más bien a una definición política y no técnica. Para esta entidad el número de casos que pudieran incluirse en el grupo de delitos de alta complejidad es razonable, aunque falta la explicación de la forma de medición de ese porcentaje. Con todo, debiera permitirse la regulación de la entrada de casos al sistema, con el objetivo de ajustar el trabajo en relación a su complejidad e importancia ${ }^{10}$.

Posteriormente, el día 2 de mayo de 2012, se presentó el mensaje del Proyecto de ley que fortalece el Ministerio Público, por parte del gobierno del expresidente Sebastián Piñera ${ }^{11}$. Este proyecto recoge las materias señaladas por el Programa de Fortalecimiento del Ministerio Público, y entre ellas, la creación de una Fiscalía Especial de Alta Complejidad.

En ese documento se reconoce la carencia institucional existente en casos de significativa complejidad y por ello se considera necesario crear una fiscalía de alta complejidad, dotada de una estructura acorde a sus necesidades de investigación para un promedio de 200 causas anuales. La competencia incluiría materias tales como drogas, corrupción, lavado de activos, trata de personas, tráfico de migrantes, terrorismo, control de armas, y delitos de que son responsables las personas jurídicas. Se trata de un organismo con competencia supraterritorial, integración

\footnotetext{
8 Ídem.

${ }^{9}$ En virtud del Programa de Servicios de Asesoría del Banco Interamericano de Desarrollo, la Dirección de Presupuestos del Ministerio de Hacienda solicitó la contratación de un estudio internacional que analice la propuesta del fortalecimiento del Ministerio Público mencionada en el párrafo anterior, con el objetivo de que presente su opinión respecto al eventual aumento presupuestario, o, en su caso, que otorgue alternativas y recomendaciones institucionales concretas para un mejoramiento de la actividad de esa institución. Grafe, Fernando (Coord.). (2011). "Nota introductoria". En: Análisis del proyecto de fortalecimiento del Ministerio Público de Chile.

${ }^{10}$ Grafe (2011), pp. 10-16.

${ }^{11}$ Se trata del Boletín No 8265-07.
} 
multidisciplinaria, y la misma jerarquía que una fiscalía regional. Con ello se sigue la línea de lo propuesto anteriormente por el Programa de Fortalecimiento del Ministerio Público ${ }^{12}$.

La técnica legislativa propuesta consistía en agregar un nuevo párrafo 4 bis al título II de la Ley Orgánica del Ministerio Público. La designación del Fiscal Jefe Especial se haría de la misma forma que en el caso de los fiscales regionales, aunque en su designación participarían las Cortes de Apelaciones de Santiago y San Miguel. Tal como se había solicitado por el Ministerio Público, implicaría la incorporación de 83 cargos $^{13}$.

En el nuevo gobierno de la presidenta Bachelet, la opción tomada por el gobierno anterior no se mantiene. Por ello, si bien la tramitación de ese proyecto de fortalecimiento continúa, mediante un boletín de indicaciones sustitutivas, se eliminan todas las referencias a la Fiscalía de Alta Complejidad, dentro de la Ley Orgánica del Ministerio Público ${ }^{14}$. En cambio, se presentó un nuevo proyecto ante el Congreso, esta vez de reforma constitucional, que continuó la discusión respecto a la gestión de los delitos complejos.

\section{Descripción del Proyecto}

\subsection{Objetivos perseguidos}

Mediante Boletín No 9608-07, de 23 de septiembre de 2014, el Ejecutivo presentó un proyecto de reforma constitucional, cuyo objetivo es crear dentro de la estructura del Ministerio Público una Fiscalía Especial de Alta Complejidad, de competencia nacional y con un funcionamiento multidisciplinario e interinstitucional.

De acuerdo a lo señalado en el mensaje del Proyecto, se advierte la necesidad de reformar la estructura del Ministerio Público, específicamente en cuanto al modo en que organiza su trabajo. Con ello, los principios de eficiencia y eficacia se cumplirán de mejor forma. Tratándose de los actos de criminalidad más graves para nuestra institucionalidad, estos principios se tornan más relevantes. Así, una persecución penal eficiente y eficaz garantiza además la protección de las víctimas de esos delitos.

Con la creación de una Fiscalía de Alta Complejidad, entonces, la persecución penal de este tipo de delitos será más eficiente y por lo tanto más efectiva. Ello implicaría entonces que con una organización exclusivamente dedicada a los delitos más graves y con personal especializado, las investigaciones serán de mayor calidad.

12 Ibíd.

13 Ibíd.

${ }^{14}$ Se trata de las indicaciones sustitutivas presentadas por el Ejecutivo el día 30 de diciembre de 2014. 
De esta forma, se obtendrán mejores pruebas y, en definitiva, se podría cumplir con el estándar necesario para obtener la condena de los sujetos que participen en la comisión de los delitos más graves.

Pero, además, se persigue un objetivo adicional, consistente en descongestionar a las fiscalías locales, permitiendo que los fiscales adjuntos puedan dedicarse a los demás casos. En la actualidad, de acuerdo al mensaje del Proyecto, estos fiscales deben suspender el resto de sus funciones cuando se les encomienda la persecución de delitos complejos. Esto produciría una distribución más eficiente del trabajo dentro de la Fiscalía, que beneficiaría también a la persecución de delitos menos complejos, que son aquellos de mayor ocurrencia en la sociedad.

\subsection{Propuestas del Proyecto}

El proyecto propone modificar la Constitución en el siguiente sentido. En primer lugar, se agregaría un nuevo artículo 85 bis. En su primer inciso, se crea la Fiscalía Especial de Alta Complejidad, con competencia nacional y a la que corresponderá ejercer permanentemente las funciones propias del Ministerio Público. Se advierte que las funciones y atribuciones de este nuevo organismo se determinarán en una ley orgánica constitucional.

En el inciso segundo, se establece que esta nueva Fiscalía será dirigida por un Fiscal Jefe Especial, designado por el Fiscal Nacional, a propuesta en terna de las Cortes de Apelaciones de la Región Metropolitana (es decir, las de Santiago y de San Miguel), conforme a lo dispuesto en el inciso tercero del artículo 86 de la Constitución ${ }^{15}$.

Por último, el inciso tercero se refiere al modo de cesación en el cargo del Fiscal Jefe Especial. Señala que "sin perjuicio de lo señalado en el artículo 89, el Fiscal Jefe Especial cesará en su cargo conforme del modo que establezca la ley orgánica constitucional respectiva”.

Se contempla que una de las facultades del Fiscal Jefe Especial sea la de proponer fiscales adjuntos, al igual como ocurre con los fiscales regionales. Por ello, se modifica el artículo 88, en el sentido de incluir al Fiscal Jefe Especial dentro de la facultad contenida en dicha norma.

El Proyecto contempla además, la modificación del artículo 89 en dos aspectos. Primeramente, se incluye al Fiscal Jefe Especial dentro de los cargos que sólo podrán ser removidos por la Corte Suprema, conforme al procedimiento que allí se establece; y en segundo lugar, se permite también que el Fiscal Nacional pueda

\footnotetext{
15 El inciso tercero del artículo 86 de la Constitución se refiere a los requisitos que deben cumplir los fiscales regionales, a saber: 1) a lo menos cinco años de título de abogado, 2) haber cumplido 30 años de edad y 3) poseer las demás calidades necesarias para ser ciudadano con derecho a sufragio. Señala además la duración del cargo (ocho años) y la imposibilidad de no poder ser designados como fiscales regionales por el período siguiente, sin perjuicio de que puedan ser nombrados en otro cargo dentro del Ministerio Público.
} 
solicitar la remoción de esta autoridad, al igual que como ocurre con los fiscales regionales.

Por último, se propone la modificación del artículo 90 para agregar al Fiscal Jefe Especial dentro de las personas que, según el artículo 81, no pueden ser aprehendidos sin orden del tribunal, salvo en el caso de crimen o simple delito flagrante y sólo para ponerlos a disposición del tribunal competente, en forma inmediata.

\section{COMEnTARio}

Nuestro ordenamiento jurídico está en lo cierto al considerar el ejercicio de la acción penal como un problema constitucional. Por supuesto, cualquier acto positivo del Estado a través del cual se manifieste el ejercicio de su potestad punitiva podrá provocar una amenaza, perturbación o privación -legítima o no- de derechos fundamentales expresamente reconocidos en la Constitución, las leyes o incluso en tratados internacionales de derechos humanos. La variedad e intensidad de esa afectación dependerá del tipo de actividad y podrá llegar a la privación de la libertad -entendida como el modo primario de ejercicio del ius puniendi ${ }^{16}-\mathrm{e}$ incluso en algunos casos, a la afectación de la vida misma ${ }^{17}$.

Sin embargo, la relevancia constitucional de la regulación de la acción penal no radica solamente en la forma como se afectan las relaciones entre el Estado y sus ciudadanos, pues esa normatividad demuestra también un problema de distribución de competencias y controles dentro del aparato estatal. De acuerdo a DíEZ-PiCAZO, se trata de un problema que no responde sólo a la -fuerte-afectación de derechos de las personas, sino que también al fundamento, la organización y los límites del poder ${ }^{18}$. Considerando esto, los problemas de diseño institucional ${ }^{19}$ que inevitablemente aparecen en la regulación de los organismos que se encargarán del ejercicio del ius puniendi son relevantes para una discusión legislativa o de reforma constitucional, como es el caso que ahora se está comentando.

\footnotetext{
${ }^{16}$ Bascuñán Rodríguez, Antonio (2007). "Derechos fundamentales y derecho penal". Revista de Estudios de la Justicia, No 9, p. 48.

${ }^{17}$ En aquellos Estados en los que no se ha derogado -todavía- la pena de muerte.

18 Díez-Picazo, Luis (2000). El poder de acusar: Ministerio Fiscal y constitucionalismo. Barcelona: Ariel, pp. 11-12.

19 La pregunta que intenta resolver la idea de "diseño institucional" se refiere a la "justa correspondencia" del objeto diseñado con su entorno, de modo que, como explica Goodin, "una institución bien diseñada, en particular, sería aquella que resulte tanto coherente en lo interno como, externamente, en armonía con el resto del orden social en el cual se inserta". Sin embargo, esa coherencia (que en definitiva es interna) debe complementarse y evaluarse frente a criterios de evaluación externos más generales, pues en ciertas ocasiones "es posible que existan buenas razones para procurar instituciones que se ajusten mal y no bien, al resto de su entorno", por ejemplo, cuando la armonía con el entorno resulta contraria a principios normativos que son justificables por sí mismos de manera independiente. Ese podría ser el caso, por ejemplo, de los derechos fundamentales. Goodin, Robert (2003). "Las instituciones y su diseño". En: Teoría del diseño institucional, Goodin, Robert (Ed.), Barcelona: Gedisa, pp. 56-59.
} 
Así, por ejemplo, uno de los problemas a los que se enfrenta toda regulación sobre esta materia es qué hacer concretamente ante la comisión de un delito. Las respuestas -desde una perspectiva de modelos comparados- podrán encontrarse en un abanico de posibilidades cuyos extremos son, por un lado, la obligatoriedad, y por otro, la discrecionalidad absoluta. Esto se traduce en la preeminencia del respeto al principio de legalidad o en la mayor posibilidad de ejercicio del principio de oportunidad, respectivamente ${ }^{20}$.

El modelo chileno sigue la tendencia en América Latina, donde la entrega de facultades de selección de casos es la respuesta que han dado las reformas al proceso penal, con el objetivo de permitir la producción de respuestas diferenciadas, la posibilidad de negociación de formas de salida más breves y sencillas con el imputado, y con ello ampliar el abanico de soluciones posibles. Sin embargo, se ha criticado la falta de análisis empíricos previos para determinar el diseño específico del principio de oportunidad, lo que lleva a concepciones muy abstractas respecto de cómo pueden solucionarse esos asuntos ${ }^{21}$.

Otro problema se refiere a la persecución de delitos de alta complejidad y cómo se organiza la institucionalidad para cumplir con ese cometido. Sin perjuicio de algunas opiniones en contrario ${ }^{22}$, existe bastante consenso en cuanto a la necesidad de contar con una organización especializada en este tipo de delitos, y por ello se ha trabajado en perspectiva comparada y desde hace varios años en esta nueva institución. Lo que se evidencia es que las imputaciones por delitos de alta complejidad tienen un impacto no sólo jurídico, sino que también político, económico y social ${ }^{23}$.

\footnotetext{
${ }^{20}$ Díez-Picazo (2000), pp. 29-32.

21 Duce, Mauricio y Riego, Cristián (2006). Desafios del Ministerio Público Fiscal en América Latina. Santiago: Centro de Estudios de Justicia de las Américas, pp. 27-28.

22 Por ejemplo, la Asociación de Fiscales se ha mostrado en contra pues esta nueva institución solo será más "burocracia", y "centralista" ya que no logrará resolver las causas de alta complejidad en regiones, pues afectaría a una cantidad muy limitada de causas. En Radio UCHILE (2014). Asociación de Fiscales cuestiona utilidad de nueva Fiscalía de Delitos de Alta Complejidad. Disponible en: <http://radio.uchile.cl/2014/12/09/ asociacion-de-fiscales-cuestiona-utilidad-de-nueva-fiscalia-de-delitos-de-alta-complejidad>. [Consulta: 13 abril 2015]. En un sentido similar, el senador Quinteros critica el centralismo del proyecto, pues aunque si bien apoya la necesidad de legislar, señala que el argumento de complejidad de los eventuales delitos investigados o la necesidad de contar con personal especializado puede llevar a una excesiva concentración de causas. Además se mostró preocupado respecto a la posibilidad que sea la connotación pública de los hechos el criterio para establecer que causas conocerá la Fiscalía. Disponible en: <http://www.soychile.cl/ Puerto-Montt/Politica/2015/02/08/303925/Rabindranath-Quinteros-cuestiona-la-creacion-de-Fiscal-de-AltaComplejidad.aspx>. [Consulta: 21 abril 2015]. Por último, a juicio del senador Baldo Prokurica, junto con argumentar que la reforma procesal penal ha sido un "fracaso", reconoce que no está convencido de que con la creación del cargo de Fiscal Jefe Especial se resuelvan los problemas reales de justicia que denuncia día a día la gente, ya que "la gente está cansada de que no se encuentre a los responsables de los delitos o que estos mismos queden en libertad". Disponible en: <http://www.baldoprokurica.cl/discusion-proyecto-deley-que-crea-fiscal-especial-de-alta-complejidad-prokurica-la-reforma-procesal-penal-es-un-sistema-fallido/>. [Consulta: 21 abril 2015].

${ }^{23}$ Duce y Riego (2006), p. 14.
} 
Además, a mi juicio, la creación de esta fiscalía ayuda a la solución de los problemas de estructura rígida cuasijurisdiccional que impone, por ejemplo, una persecución territorial de los delitos. En efecto, a través de una competencia nacional se puede derivar una forma de asignación menos rígida de casos para los fiscales, lo que en este tipo de casos resulta crucial. Esta nueva organización fortalece el trabajo en equipo, que se ha visto un poco abandonado a nivel latinoamericano, y es también una manifestación de liderazgo estratégico, que es beneficioso para la actualización de la Fiscalía. En definitiva, se trata de una manifestación de una decisión de abandonar el paradigma tradicional asociado a la forma de trabajo judicial ${ }^{24}$.

Ahora bien, como se puede apreciar, la principal característica de este proyecto, y que constituye una innovación respecto de las propuestas anteriores, es que constitucionaliza la Fiscalía de Alta Complejidad. Este cambio en el devenir legislativo del proyecto nos lleva a un problema de ingeniería constitucional, específicamente a la pregunta de si es necesaria esa constitucionalización.

En mi opinión, existen buenas razones para esta opción constitucional. Por supuesto, en términos de coherencia del sistema normativo, se trata de una alternativa que se adecua a la regulación actual del Ministerio Público, pues la estructura de las fiscalías regionales tiene una jerarquía constitucional, y como se ha explicado, esta fiscalía especial tendrá el mismo grado que esos organismos.

Pero con esta opción se logra algo que va más allá. Como es sabido, el diseño constitucional chileno implica un aumento de requisitos respecto a la ley para su modificación. Es cierto, como explica ATRIA, que en ciertos casos una norma constitucional sólo persigue una obstrucción mañosa del ejercicio de la mayoría -lo que en todo caso resulta más evidente en las leyes orgánicas constitucionales dictadas los días antes del término de la dictadura- ${ }^{25}$, pero a mi juicio, en otros resulta justificable que una institución sea blindada a través de su inclusión en la Constitución, puesto que esa incorporación es coherente con las características que han sido pensadas para ese órgano.

En otras palabras, si de lo que se trata es evitar conductas corruptas y arbitrarias, entonces, la pregunta de diseño institucional consiste en cómo hallar mecanismos procesales y organizativos que permitan esa restricción ${ }^{26}$. Así, a través de una norma constitucional es más probable asegurar algunas características importantes del órgano en cuestión.

Por ejemplo, considerando que habitualmente las normas constitucionales posean un quórum de reforma más alto que la ley, tiene mucho sentido que la regulación principal del Poder Judicial y del Ministerio Público sea a través de

\footnotetext{
${ }^{24}$ Estas ventajas se pueden entender como avances en el diseño y gestión del Ministerio Público, en comparación con sus pares latinoamericanos. Vid. DuCE y RIEGO (2006), pp. 40-42, 48, 60 y 68.

${ }^{25}$ Atria Lemaître, Fernando (2013). La Constitución Tramposa. Santiago: LOM Ediciones, pp. 44-53.

${ }^{26}$ Diez-Picazo (2000), p. 25.
} 
normas de rango constitucional, pues así es más probable asegurar la independencia de estos órganos ${ }^{27}$. Esto implica que un órgano dotado de esta autonomía constitucional no podrá ser subordinado en el cumplimiento de sus funciones, a las decisiones de los principales -y tradicionales- órganos del Estado, especialmente de aquellos dependientes de la Administración, ya sea por leyes o por otras normas infraconstitucionales ${ }^{28}$.

Así, la creación de una fiscalía de alta complejidad a nivel constitucional se traduce en una mayor autonomía del Ministerio Público, y ello es relevante, pues asegura que este organismo tenga el espacio institucional necesario para que pueda cumplir sus funciones y objetivos de acuerdo con el nuevo sistema procesal penal. Sin embargo, se debe reconocer que esa independencia no se puede traducir en aislamiento, pues es esencial que existan mecanismos de coordinación con los demás órganos del Estado ${ }^{29}$.

En conclusión, además de la coherencia normativa que se persigue con este proyecto, resulta positivo incorporar la Fiscalía de Alta Complejidad en la Constitución. Con ello se dificultaría la posibilidad de suprimir este nuevo organismo en el futuro, o se fortalecería este organismo frente a los riesgos de utilización de la acción penal en un contexto de excesiva discrecionalidad.

Esta idea adquiere mayor importancia hoy, considerando que en los últimos meses se ha visto la importancia de contar con un Ministerio Público fuerte, que sea capaz de investigar situaciones que involucran a quienes ejercen el poder político o quienes poseen poder económico, y posteriormente ejercer sus atribuciones constitucionales ante la justicia penal. De esta forma, la propuesta del proyecto que se está comentando puede ser entendida como una mejor solución organizativa para evitar problemas de corrupción o conflictos de interés, y en definitiva como una buena decisión para lograr mejores estándares de eficiencia y eficacia.

De lo que se trata entonces es de perfeccionar el Ministerio Público a través de este tipo de iniciativas, pues de esta forma se puede asegurar que cumpla el fin para el que fue creado como complemento de la reforma a la justicia de gabinete y la manipulación del poder penal. A través de este tipo de medidas el Ministerio Público puede efectivamente llegar a ser "un medio de liberación ciudadana y no como instrumento de represión autoritaria” ${ }^{30}$.

\footnotetext{
27 Vid. Atria Lemaître, Fernando (2007). "La improbabilidad de la jurisdicción”. En: La judicatura como organización, ATRIA, Fernando y Couso, Javier (Edits.) Santiago: Expansiva, pp. 33-55.

${ }_{28}$ ZúNiga Urbina, Francisco (2007). "Autonomías constitucionales e instituciones contramayoritarias (a propósito de las aporías de la democracia constitucional)”. Ius et Praxis, Vol. 13, No 2, pp. 230-231.

${ }^{29}$ Duce y Riego (2006), pp. 27 y 38.

${ }^{30}$ Roxin (1993), p. 39.
} 